\title{
Insights on the epigenetic mechanisms underlying pulmonary arterial hypertension
}

\author{
R.C.P. Luna ${ }^{1 *}$, Y. de Oliveira ${ }^{1 *}$, J.V.C. Lisboa ${ }^{1}$, T.R. Chaves ${ }^{1}$, T.A.M. de Araújo ${ }^{1}$, E.E. de Sousa ${ }^{1}$, \\ M. Miranda Neto ${ }^{1}$, L. Pirola ${ }^{2}$, V.A. Braga ${ }^{3}$ and J.L. de Brito Alves ${ }^{1,3}$ \\ ${ }^{1}$ Departamento de Nutrição, Centro de Ciências da Saúde, Universidade Federal da Paraíba, João Pessoa, PB, Brasil \\ ${ }^{2}$ INSERM U1060, Lyon 1 University, Oullins, France \\ ${ }^{3}$ Departamento de Biotecnologia, Centro de Biotecnologia, Universidade Federal da Paraíba, João Pessoa, PB, Brasil
}

\begin{abstract}
Pulmonary arterial hypertension (PAH), characterized by localized increased arterial blood pressure in the lungs, is a slow developing long-term disease that can be fatal. PAH is characterized by inflammation, vascular tone imbalance, pathological pulmonary vascular remodeling, and right-sided heart failure. Current treatments for PAH are palliative and development of new therapies is necessary. Recent and relevant studies have demonstrated that epigenetic processes may exert key influences on the pathogenesis of $\mathrm{PAH}$ and may be promising therapeutic targets in the prevention and/or cure of this condition. The aim of the present mini-review is to summarize the occurrence of epigenetic-based mechanisms in the context of PAH physiopathology, focusing on the roles of DNA methylation, histone post-translational modifications and non-coding RNAs. We also discuss the potential of epigenetic-based therapies for $\mathrm{PAH}$.
\end{abstract}

Key words: Epigenetic; Pulmonary arterial hypertension; DNA Methylation; Histone acetylation; miRNAs

\section{Introduction}

Pulmonary arterial hypertension $(\mathrm{PAH})$ is a severe and multifactorial disease with a high incidence worldwide (1). The incidence of $\mathrm{PAH}$ ranges from 2 to 7.6 cases per million adults per year, while its prevalence varies from 11 to 26 cases per million adults (2-4). PAH is defined as mean pulmonary artery pressure above $25 \mathrm{mmHg}$ and pulmonary artery occlusion pressure lower than $15 \mathrm{mmHg}$. $\mathrm{PAH}$ is categorized as idiopathic (iPAH), inheritable, toxinor drug-induced, or linked to other pathological conditions, such as human immunodeficiency virus (HIV) infection, portal hypertension, congenital heart disease, or schistosomiasis.

Despite considerable advances in the understanding of the pathophysiology, diagnosis, and treatment of $\mathrm{PAH}$, the molecular mechanisms underlying the PAH remain unclear. Physiopathological alterations in patients with $\mathrm{PAH}$ have been related to age, sex, and the presence of co-morbidities, and they collectively contribute to the patient's survival (5).

Recent findings have indicated that epigenetic modifications may be associated to the pathogenesis of $\mathrm{PAH}(6,7)$. Evidence shows that DNA methylation, histone post-translational modifications, and micro-RNA (miRNA)associated gene silencing are found in both human and animal models of PAH (8). For example, histone deacetylation and miRNAs dysregulation are observed in the hyper-proliferation of pulmonary artery smooth muscle cells (PASMCs) (7). Additionally, DNA cytosine methylation can silence genes through a process that leads to the compaction of the chromatin structure or by acting directly on the DNA promoter region (9). Accordingly, previous studies have shown that DNA hypermethylation in the superoxide dismutase 2 (SOD2) genomic region can lead to reduced SOD2 expression in PASMCs and contribute to hyper-proliferation of these cells in PAH patients $(10,11)$.

To highlight these new insights, we discuss here the current advances in our understanding of how epigenetic dysregulations are associated with $\mathrm{PAH}$, and how targeting such defects may open the way to innovative therapies.

\section{Physiopathology of PAH}

The pathogenesis of $\mathrm{PAH}$ is complex and still has not been completely elucidated. Thus, a better understanding of disease pathogenesis is essential to identify new targets for therapy. PAH is characterized by augmented vasoconstriction, vascular obstruction, inflammation,

Correspondence: J.L. de Brito Alves <jose_luiz_61@hotmail.com>

${ }^{*}$ These authors contributed equally to this work. 
fibrosis, vascular stiffening, endothelial dysfunction, and right ventricular failure (12).

Abnormal pulmonary vascular reorganization, including excessive vascular proliferation and resistance to apoptosis of pulmonary artery smooth muscle cells (PASMCs) within the vascular wall, contributes to reduction of arterial compliance and increased vascular resistance and blood pressure, resulting in right-side heart failure and premature death. Patients with PAH exhibit multiple nonspecific symptoms, with fatigue, weakness, dizziness, and progressive shortness of breath on exertion being often reported by patients (12).

Several underlying mechanisms leading to excessive proliferative and reduced apoptosis in PASMCs have been previously elucidated. For example, increased cytosolic calcium via activation of store-operated $\mathrm{Ca}^{+2}$ channels or down-regulation of voltage-gated potassium channels, such as Kv1.5, facilitate the contractile, hyperproliferative, and anti-apoptotic phenotype of PASMCs in PAH (13). In addition, mitochondrial dysfunction, including a metabolic shift from glucose oxidation toward uncoupled aerobic glycolysis, unregulated glycolysis and mitochondrial fragmentation, and membrane hyperpolarization support rapid proliferation and resistance to apoptosis in PASMCs (14).

The importance of cardiac dysfunction in the right ventricle (RV) in $\mathrm{PAH}$ is attracting increasing interest due to its critical association with the morbidity and mortality of the disease. An alteration of the autonomic balance, characterized in particular by a dysfunction of cardiac variability and a reduced parasympathetic sensitivity (15), is associated with a poor prognosis in PAH patients (16). In addition, hyperactivation of the sympathetic nervous system has been demonstrated in clinical and experimental studies as one of the main factors associated with autonomic dysfunction in PAH (17). Sympathetic hyperactivation is characterized by an increase in the intensity and frequency of electrical depolarizations of the sympathetic nerve and by an increase in the plasmatic levels of catecholamines, promoting constriction of peripheral blood vessels, increased vascular resistance, and consequent increase in baseline blood pressure levels. Because of this, circulating catecholamines are augmented in $\mathrm{PAH}$ patients with RV failure (18). In addition, endothelial dysfunction has been observed in the development and progression of vascular pathology in $\mathrm{PAH}$. Patients with $\mathrm{PAH}$ exhibit pulmonary endothelial dysfunction and decreased nitric oxide (NO) bioavailability, characterized by a reduced endothelial NO synthase (eNOS) expression, and low synthesis and release of $\mathrm{NO}$, the main vasodilator substance produced by the endothelium of the pulmonary vessels (19).

\section{Epigenetic mechanisms}

The term epigenetics was originally proposed by Conrad Waddington (1942), to describe of the occurrence of biologically relevant processes resulting from the interplay between the genome (and its functional units: the genes) and the environment, which lead to different phenotypic manifestations (20). In other words, epigenetics refers to the manifestation or transmission of specific characters whose information is not contained within the DNA 4 base code. The main epigenetic mechanisms that in mammalian cells contribute to the regulation of gene expression include DNA methylation, histone post-translational modifications (such as methylation, acetylation, phosphorylation), and ubiquitination (21). More recently, non-coding RNAs have been also demonstrated to be involved in the post-transcriptional regulation of gene expression in multicellular organisms (22).

Cytosine DNA methylation, taking place on cytosines within the CpG dinucleotide sequence, is a process catalyzed by DNA methyltransferases (DNMTs) enzymes, and is a key epigenetic mechanism associated to gene expression modulation. It has been proposed that $\mathrm{CpG}$ islands, found in gene promoter and other genomic regulatory relevant regions, when hyper-methylated, lead to gene silencing (23).

In addition to cytosine methylation, post-translational modifications taking place in the histone subunits composing the histone octamer also contribute to the definition of gene expression patterns. Histones are highly evolutionarily conserved proteins, which are essential in the composition of the nucleosome of eukaryotic chromatin. The histone octamer is composed of dimers of each of four central core histones ( $\mathrm{H} 2 \mathrm{~A}, \mathrm{H} 2 \mathrm{~B}, \mathrm{H} 3$, and $\mathrm{H} 4)$ (24). DNA is wrapped around the histone octamer, and collectively, the histone octamer and the associated DNA form the nucleosome: the basic repeating unit of chromatin. Nucleosomes are "sealed" externally by histone H1. Histone acetylation is a reversible and dynamic epigenetic mechanism determined by the balance between histone acetyltransferases (HATs) and histone deacetylases (HDACs). In general, the deacetylation process is associated with condensed chromatin (heterochromatin) and transcriptional repression.

miRNAs act as important regulators of gene expression as part of the epigenetic machinery. Distinct biotypes of extracellular RNA have been detected in human circulation in the form of small regulatory non-coding RNAs (sRNAs). Typically, sRNAs range from 15 to 200 nucleotides, with miRNAs being the most widely studied in mammals (25).

\section{DNA methylation}

DNA methylation, one of the most widely studied epigenetic mechanisms, is a biochemical reaction of covalent addition of a methyl $\left(\mathrm{CH}_{3}\right)$ group to cytosine residues, usually within $\mathrm{CG}$ dinucleotides enriched in genomic regions named $\mathrm{CpG}$ islands. DNA methylation is accomplished by DNMTs (26). DNMTs are thus involved in de novo DNA methylation and maintenance of genome methylation (27). Four main variants of DNMTs are found in humans, 
namely DNMT1, DNMT3A, DNMT3B, and DNMT3L, to mediate the methylation process. DNMT1 serves to maintain the existing methylation patterns. DNMT3A and DNMT3B, on the other hand, regulate the de novo methylation, while DNMT3L functions as a unique co-factor in the methylation of imprinted genes in gametic cells (28). In general, DNA methylation promotes condensation of chromatin structure, leading to the silencing or suppression of gene expression.

\section{Histone post-translational modifications (PTMs)}

Enzyme-mediated acetylations and methylations occurring on histones do not constitute the only possible PTMs taking place on histones. Indeed, while acetylation and methylation are the most studied phenomena, histones are also submitted to phosphorylation, sumoylation, ubiquitination, and ribosylation. These modifications appear in specific amino acids: acetylation on lysines $(\mathrm{K})$, methylation on lysines and arginines (R), phosphorylation on serines (S) and threonines ( $T$ ), ubiquitylation, and sumoylation and ribosylation on lysines (29).

Collectively, these PTMs have an influence on the structure and the level of condensation of the chromatin and are part of a "histone code" at the basis of gene regulation (30). Histone acetylation and deacetylation play an important part in the nucleosome modifications process. They are caused by acetylation and deacetylation complexes and yield a covalent modification of the nucleosome by changing the histones tail conformation by adding and removing acetyl groups from the amino termini of the four core histones. Acetylation and deacetylation are mediated by HATs and HDACs, respectively. Acetylation, mediated by HATs, is the reversible reaction where one acetyl group is transferred from an AcetylCoA to the lysine e-amino group. The removal of the acetyl moiety is made by HDACs, with the production of $\mathrm{H}_{2} \mathrm{O}$. Acetylation is mostly linked to transcriptional activation.

Histone acetyltransferases (HATs). HATs can be separated into two groups: Type $A$ located in the nucleus and acetylating histones, in the timing needed for transcriptional activation, and Type B located in the cytoplasm, responsible for histone acetylation during replication and before the chromatin's assemblage. HATs are responsible for histone acetylation, but can also acetylate other nonhistones proteins in various nuclear and cytoplasmic pathways (31). Among the most active HATs in mammals are found CAMP response-element binding protein (CREB) binding protein (CBP), p300, p300/CREB binding proteinassociated factor (PCAF), and HIV Tat interactive 60-kDa protein (Tip60). Specific HATs recruited by steroid receptors exist also, such as steroid receptor coactivators 1 and 3 (SRC-1 and -3) (31).

Histone deacetylases (HDACs). HDAC activity was first discovered in yeast. The use of trapoxin, an antitumor cyclic tetrapeptide, showed increased histone acetylation, and led to the identification of the protein responsible: the first histone deacetylase. HDACs can be separated into 3 different groups based on their similarities with the yeast histone deacetylase: $i$ ) class I HDACs (HDAC 1, 2, 3 , and 8 ) located in the nucleus and similar to the yeast RPD3 protein, ii) class II HDACs (HDACs 4, 5, 6, 7, 9, and 10) present in both nucleus and cytoplasm and similar to the yeast HDA1 protein, and iii) class III HDACs (Sirtuins 1-7) analogous to the yeast Sir2 proteins. HDACs play a major role adjusting acetylation and deacetylation levels of chromatin (32).

Sirtuins. A particular HDAC class, sirtuins are a specific class of HDACs that are dependent on nicotine adenine dinucleotide $\left(\mathrm{NAD}^{+}\right)$for their activity. They regulate numerous activities like cell division, transcription, metabolism, stress damage, and aging. Seven sirtuins exist with specific locations in the cell: Sirt1, 6 and 7 are in the nucleus, Sirt2 in the cytosol, and Sirts3, 4, and 5 in the mitochondria (33).

Histone methylation. Histone methylation is a transcriptionally promoting, or repressing PTM. Methylation concerns lysine and arginine residues. Contrary to acetylation, methylation marks can be multiple. Arginines can be unmethylated, monomethylated, or dimethylated. Furthermore, arginine dimethylation can generate asymmetric dimethylarginines and symmetric dimethylarginines (34). Each lysine residue can be mono-, di- or trimethylated. Histone lysine methylation appears to occur preferentially on $\mathrm{H} 3$ and $\mathrm{H} 4$. Methylation is catalyzed by histone methyltransferases (HMTs). The methyl-transfer reaction uses s-adenosylmethionine (SAM) as a methyl donor; in the same way, HATs use acetyl groups derived from acetylCoA (35). Histone methylation is a more permanent mark than acetylation. Indeed, histone acetylation can occur all along the cell cycle whereas histone methylation is associated to heterochromatin formation with methylation levels peaking in the G2 phase following DNA replication and histone re-deposition.

\section{Histone methyltransferases}

Methylation is orchestrated by HMTs. HMTs responsible for the arginine methylation are called protein arginine methyltransferases (PRMTs), and 11 isoforms, divided into 3 groups, type I, II, and III, exist in mammals (36). In the same way, methyltransferases responsible for the specific methylation of lysines are called lysine methyltransferases (KMTs) and are divided into six families based on the structural particularity of their catalytic SET domain (37).

Histone demethylases (HDMs). As mentioned above, methylation was thought to be an irreversible/permanent epigenetic mark, a belief derived from the high thermodynamic stability of the $\mathrm{N}-\mathrm{CH}_{3}$ bond. The understanding of the methylation regulation changed with the discovery of one histone demethylase, LSD1. Later, many enzymes regulating histone demethylation were discovered. Demethylation mechanisms differ depending on the amino acid carrying the methylation, lysine or arginine, and the methylation profile, one to three methyl groups. Two types 
of histone demethylases exist: a flavin adenine dinucleotide (FAD)-dependent amine oxidase and Fe (II) and $\alpha$-ketoglutarate-dependent dioxygenase. For the first type, which includes the amine oxidase LSD1, demethylation requires FAD as a cofactor during the removal of a methyl group and produces hydrogen peroxide and formaldehyde (38). In the second type, demethylation by an irondependent and alpha-ketoglutarate-dependent oxidation reaction mechanism as found in yeast is conserved in eukaryotes. The enzyme, containing a JmjC domain, is capable of demethylating DNA, producing formaldehyde and succinate as reaction products.

\section{Ubiquitination}

The ubiquitin-proteasome system (UPS), a major protein quality and quantity control system, is a post-translational mechanism particularly interesting from both structural and functional viewpoints, involved in the regulation of many cellular processes including protein degradation, gene expression, signaling transduction, and apoptosis (39). Ubiquitin is a 76-amino acid protein ubiquitously distributed in all tissues of eukaryotic organisms. In this process, the C-terminal carboxyl group of ubiquitin becomes attached to the $\epsilon$-amine of a lysine residue of the substrate protein through an isopeptide bond. Ubiquitination is a multi-step and reversible process that involves a cascade of three essential enzymes: ubiquitin-activating enzymes (E1), ubiquitin-conjugating enzymes (E2), and ubiquitin ligases (E3). E1 and E2 enzymes prepare ubiquitin for conjugation. E3 enzymes recognize the specific substrate and catalyze the transfer of activated ubiquitin to the substrate. Recently, the activity of an E4 enzyme has been described as catalyst for the conjugation of additional ubiquitin monomers to form polyubiquitin chains, usually through lysine 48 (K48) linkages (40). The processes of ubiquitination and de-ubiquitination are controlled with high specificity; however, dysfunctions in this complex are implicated in many human diseases (40).

\section{Non-coding RNAs}

Non-coding RNAs (ncRNAs) are the transcription products of non-coding genes (i.e., lacking the ability to be translated into proteins). Non-coding RNAs include tRNAs, rRNAs, snoRNAs, microRNAs, siRNAs, snRNAs, exRNAs, piRNAs, and scaRNAs and the long ncRNAs (41). The development of next-generation sequencing technologies identified thousands of ncRNAs. These ncRNAs can be divided into two main classes: small ncRNAs $(<200$ nucleotides long), which include microRNAs (miRNAs), piwiinteracting RNAs (piRNAs), and small-interfering RNAs (siRNA), their main function is to modulate gene expression through direct binding to coding or non-coding sequences of mRNAs; and long-non-coding RNAs (IncRNAs) (>200 nucleotides long), which include natural antisense transcripts, small nucleolar RNAs (snRNA), and other types of IncRNAs. Specifically, miRNAs are post-transcriptional regulators, piRNAs can modulate DNA methylation and transposon repression, and siRNAs are known as short interfering RNA or silencing RNA, similar to miRNA, and operating through the RNA interference (RNAi) pathway. In addition, IncRNAs are epigenetic regulators of transcription, the snRNAs are mainly involved in nucleotide modification of ribosomal RNA, and circular RNAs (circRNAs) are miRNA sponging and RNA polymerase II regulators (41).

\section{DNA methylation and PAH}

It has been suggested that down-regulation of SOD2 can activate the hypoxia-inducible factor 1 alpha (HIF-1 $\alpha$ ) and create a pseudo-hypoxic environment favorable to a glycolytic metabolic state and harmful to oxidative metabolism in PASMCs of fawn-hooded rats. Studies have shown that the hypermethylation mechanisms in $\mathrm{CpG}$ islands mediated by DNMT1 and DNMT3B contribute to the down-regulation of SOD2 mRNA in PAH $(10,11)$. These alterations may be able to enhance the proliferation of the PASMCs in PAH.

It is reasonable to suggest that DNA methyltransferase inhibitors, by decreasing DNA methylation on the SOD2 gene locus and consequently favoring the SOD2 gene and protein expression, may be able to alleviate PASMCs proliferation and consequently SOD2 down-regulation in $\mathrm{PAH}$. Studies conducted on the fawn-hooded rat, which develops PAH spontaneously, have demonstrated that treatment with 5-aza-2'-deoxycytidine (a DNMT inhibitor) or with MnTBAP (a mimetic of SOD2), at a dose of $10 \mathrm{mg} / \mathrm{kg}$ for 2 weeks, was capable of increasing SOD2 expression while reducing the proliferative state of PASMCs, resulting in alleviated pulmonary arterial hypertension $(10,42)$. Therefore, the understanding of the relationship between DNA methylation and SOD2 expression might be an important step towards better elucidation of the pathophysiologic alterations in $\mathrm{PAH}$ and possibly the development of a new therapeutic target for this disease. In addition, future studies will need to examine whether HIF-1 $\alpha$ inhibitors are able to exhibit beneficial results under the PAH condition.

Heritable forms of PAH represent approximately $6-10 \%$ of all PAH. From a genetic basis, the most recognized genetic variants linked with $\mathrm{PAH}$ occur in type 2 bone morphogenetic protein receptor 2 (BMPR2). BMPR2 mutations are responsible for the etiology of approximately $80 \%$ of patients with familial PAH and $30 \%$ idiopathic PAH $(43,44)$. Thus, a BMPR2 gene mutation increases the chance of developing $\mathrm{PAH}$. Another important point is that BMPR2 mutations are considered to be permissive of disease and require additional genetic, epigenetic, or environmental influences for the development of PAH in individuals with mutations (2). Low BMPR2 protein expression or impaired BMPR2 signaling in lung tissue and endothelial cells have been shown to promote accelerated cell proliferation and facilitate the development of PAH (45). A study analyzing whether alterations in DNA methylation 
pattern could be associated to BMPR2 mutations in 28 patients with $\mathrm{iPAH}$ and 27 patients diagnosed with $\mathrm{PAH}$ associated with other diseases found no difference in the methylation $\mathrm{CpG}$ islands of BMPR2 promoter region between the $\mathrm{PAH}$ patients and healthy control subjects (46). On the contrary, a recent study demonstrated that hypermethylation in the BMPR2 promoter does occur in patients with heritable pulmonary arterial hypertension, resulting in down-regulation of BMPR2 expression (47). Together, these findings show that DNA methylation mechanisms involved in PAH are complex and unmistakable, take place on multiple genes, and are not the only mechanisms associated with all forms of $\mathrm{PAH}$. Therefore, there is a need to further explore cytosine methylation mechanisms in $\mathrm{PAH}$ as potential targets for future new therapies.

\section{Histone acetylation and PAH}

Recent findings have shown that histone acetylation is likewise important in cardiopulmonary remodeling. However, a satisfactory understanding of the mechanism(s) by which histone acetylation dysregulation impacts pulmonary remodeling in $\mathrm{PAH}$ will require more research (48).

Talati and colleagues observed that histone $\mathrm{H} 1$ expression was reduced in the pulmonary artery and PASMCs of patients with $\mathrm{IPAH}$. The authors suggested that the reduced $\mathrm{H} 1$ expression could contribute to a less condensed chromatin pattern and consequently facilitate the activation of transcriptional pathways contributing to $\mathrm{PAH}$ (49).

Histone acetylated lysines, under the control of the opposite action of HATs and HDACs, are recognized by bromodomain and extra-terminal (BET) proteins. BET proteins binding to acetylated histones promote transcriptional elongation and upregulation of genes involved in cell proliferation, apoptosis, and inflammation (50).

HAT activity and the HAT:HDAC ratio have been reported to significantly increase in the lungs of patients with iHAP. High HAT activity has also been linked to elevated expression levels of BET proteins under the PAH condition (50). BET proteins are essential for the activation of inflammatory transcription nuclear factor kappa-light-chain-enhancer of

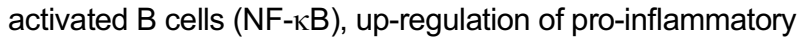
genes, alteration in the proliferation/remodeling of vascular endothelial cells, and appear to play a direct role in the $\mathrm{PAH}$ pathogenesis (51).

Interestingly, the inhibition of BETs in primary human pulmonary microvascular endothelial cells (HPMECs) induced a significant reduction in inflammation and pulmonary remodeling (50). Thus, BET inhibition is another promising target for future therapies against $\mathrm{PAH}$.

An excessive accumulation of reactive oxygen species (ROS), increased expression of NADPH oxidase and downregulation of antioxidant enzymes also has been reported in PAH. In addition, a defect of the histone acetylation process has been observed in oxidative stress pathways (52).
Of note, Chen and colleagues found that HDAC inhibitors potently reduce transcription of NADPH oxidases and ROS production and ameliorate $\mathrm{PAH}$ in monocrotaline (MCT) rat model (53).

SOD3, an extracellular isoform of the superoxide dismutase, is an important antioxidant enzyme in the vasculature system. Experimental and clinical studies have reported that SOD3 expression is reduced in PAH $(54,55)$. Nozik-Grayck and colleagues investigated whether aberrant DNA methylation and/or histone deacetylation could be the reason for reduced SOD3 expression in $\mathrm{IPAH}$. The authors demonstrated that DNA methylation was not responsible for SOD3 down-regulation in PASMC of iPAH patients (56). Additionally, HDAC and HAT activities were similar in the PASMC of healthy subjects and $\mathrm{PAH}$ patients. However, it was observed that treatment with selective class I HDAC inhibitors increased SOD3 expression in PASMC of iPAH patients. Thus, targeting of HDACs with HDAC inhibitors (HDACi) could represent a potential therapeutic approach for iPAH, at least in part, because of the HDACi-induced SOD3 expression in PASMC (56).

Similarly, treatment with valproic acid, a class I HDAC inhibitor, at $300 \mathrm{mg} / \mathrm{kg}$ during 5 weeks, has also been shown to reverse the development of severe pulmonary arterial hypertension in MCT and chronic hypoxia rat models (57). Based on those findings, it is reasonable to propose that HDACi may be useful in the treatment of PAH.

\section{Ubiquitination and proteasome activity in PAH}

Although the precise mechanism(s) still need to be fully elucidated, recent studies have demonstrated that the ubiquitin-proteasome complex might be involved in PASMCs proliferation in $\mathrm{PAH}$, and consequently might represent a new target for treatment of PAH $(58,59)$. It has been demonstrated that intersectin-1s (ITSN-1s), a multidomain adaptor protein regulating endocytosis, cytoskeletal rearrangements, cell signaling, and the ubiquitination process is reduced in $\mathrm{PAH}(60)$.

In addition, it has been shown that proteasome inhibitors can suppress the growth of pulmonary PASMCs and consequently might be useful for treating PAH (59). Using bortezomib (BTZ), the first proteasome inhibitor to be approved by the FDA, pulmonary vascular remodeling was successfully reversed in PAH rats (61). However, BTZ caused cardiac apoptosis in both the RV and the left ventricle (LV) in PAH rats, limiting their clinical applicability. Carfilzomib (CFZ), another proteasome inhibitor, administration in combination with vasodilators and cardioprotectants has been demonstrated as an effective therapy for the treatment of PAH. CFZ was more effective in killing human pulmonary vascular cells than BTZ (62). The mechanism of proteasome inhibition involves the active role of ubiquitin in promoting apoptosis as well as autophagy in PASMCs in PAH (62). 


\section{Role of non-coding RNAs in PAH}

Traditionally, studies of gene expression and epigenetics have been focused on proteins that act as transcription factors and enzymes modulating histones post-translational modifications and DNA methylation. More recently, cumulative evidence suggested the involvement of RNA molecules as an additional regulatory player contributing to the determination of chromatin structure.

It is well known that the primary cellular mechanism underlying vascular remodeling under the $\mathrm{PAH}$ condition is the excessive proliferation of PASMCs (63). It has also been suggested that PASMC proliferation may be associated with aberrant microRNA signatures, which might provide some insights into the pathogenesis of PAH $(64,65)$.

miRNAs have been described as playing essential roles in genic modulation and various cellular process, such as proliferation, differentiation, and apoptosis (66). There is growing evidence that a set of miRNAs is involved in the process of angiogenesis and vascular remodeling, which are prognostic events in the pathogenesis of $\mathrm{PAH}(66-68)$. Thus, investigation and understanding of the miRNA dysregulation events taking place in $\mathrm{PAH}$ may lead to the uncovering of useful biomarkers and/or new therapeutic targets for the treatment of PAH (69).

Previous reports have shown that a number of miRNAs, such as miR21, miR-103/107, miR-140-5p, miR-199a-5p, miR-223, let-7a-5p, miR-26b-5p, miR-27b-3p, miR-199a-3p, and miR-656, act together to control PASMCs proliferation in PAH both in cell culture and rat models (Table 1).

MiR-21 expression has been shown to be augmented in hypoxia-induced PASMCs proliferation. Interestingly, when miR-21 is inhibited (anti-miR-21), cell proliferation is reduced in primary PASMCs cell cultures derived from patients with $\mathrm{PAH}$, demonstrating that miR-21 plays an important function in PASMCs proliferation (70).

Hypoxia, a common stimulus for $\mathrm{PAH}$, decreased miR-98 expression and augmented endothelin-1 (ET-1) levels in the lungs of mice. Pharmacological activation of peroxisome proliferator-activated gamma receptor (PPAR $\gamma$ ) with rosiglitazone restored miR-98 levels, reducing ET-1 and the proliferation of pulmonary artery endothelial cells (71).

Recently, Deng and colleagues demonstrated that miR-103/107 also regulates the proliferation of PASMCs under PAH conditions in a rat model. Down-regulation of miR-103/107 was linked to activation of HIF-1 $\beta$-dependent signaling pathways, which resulted in enhanced proliferation of PASMCs and vascular remodeling in PAH (72).

Regarding the role of miR-127a, it has been demonstrated that miR-127a is up-regulated in the lungs of a hypoxiainduced PAH experimental model. This up-regulation was linked to reduced PPAR gamma levels and pulmonary artery endothelial cell hyper-proliferation (73). Recently, the mechanism by which miR-127a/b is linked to PPAR $\gamma$ downregulation and PASMCs hyper-proliferation has been better

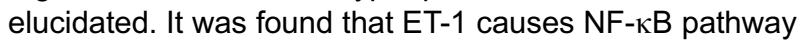

activation and subsequent miR-127a/b up-regulation, which leads to post-transcriptional suppression of PPAR $\gamma$ expression and controls the proliferation of PASMCs (74).

MicroRNA-135a has also been associated with $\mathrm{PAH}$. miR-135a levels were found to be up-regulated in the lung of an experimental mouse model displaying PAH (75). A recent study investigated whether miR-135a could influence BMPR2 expression in an experimental mouse model of PAH. Lee and colleagues demonstrated that miR-135a was significantly increased and BMPR2 significantly decreased, suggesting that BMPR2 may be an important target of miR135a in $\mathrm{PAH}$. Additionally, the study demonstrated that treatment with antagomiR-135a for three weeks, improved right ventricular systolic pressure and right ventricular hypertrophy and augmented both mRNA and protein expression of BMPR2 (75).

Hypoxia leads to upregulation of mitofusin 1 (MFN1), a mitochondrial fusion protein, both in vivo and in vitro. MFN1 is involved in hypoxia-induced PASMCs proliferation in PAH (76). Regarding the role of miR-140 on MFN1 expression in $\mathrm{PAH}$, up-regulation of $\mathrm{miR}-140$ has been shown to be linked to reduced MFN1 expression in the hypertrophic right ventricles of PAH rats (76).

Previous reports have demonstrated that the expression of miR-140-5p was reduced in experimental models and in patients with PAH $(77,78)$. Some authors have proposed that SMAD-specific E3 ubiquitin protein ligase 1 (SMURF1) could be a key regulator for miR-140-5p targets and bone morphogenetic protein (BMP) signaling. This indicates that reduced miR-140-5p expression is associated to increased pulmonary vascular SMURF1 protein expression and reduced BMP signaling in patients with $\mathrm{PAH}$ and that inhibition of SMURF1 provides a potential mechanism by which BMP signaling may be augmented for therapeutic benefit (77).

Interestingly, Dnmt1 is also a potential target of miR-140$5 p$. Reduced miR-140-5p contributes, at least in part, to Dnmt1 overexpression and consequently to down-regulation of SOD2 expression and hyper-proliferation of PASMCs in $\mathrm{PAH}$. Furthermore, increased miR-140-5p inhibits proliferation and promotes apoptosis and differentiation of PASMCs under hypoxic conditions (78).

MiR-204 attenuation has been shown to promote upregulation and sustained expression of the Runt-related transcription factor 2 (RUNX2) and HIF1 $\alpha$ in PAH. These alterations are associated with aberrant proliferation and resistance to apoptosis in PASMCs in a subset of patients with PAH (79).

MiR-223 has been primarily described in hematopoietic cells and some tumoral processes (80). Recent studies, however, have also investigated its function in $\mathrm{PAH}$ (81). Expression of miR-223 is down-regulated in the human PAH lung, distal pulmonary arterioles, and PASMCs (82). Similarly, in the hypoxia-induced PAH model, miR-223 is down-regulated in PASMCs. When miR-223 was overexpressed in hypoxia-induced PAH model, there was attenuation in pulmonary arterial pressure $(81,82)$. 
Table 1. Non-coding RNAs involved in pulmonary arterial hypertension (PAH) development.

\begin{tabular}{|c|c|c|c|c|}
\hline miRNAs & $\begin{array}{l}\text { Expression } \\
\text { in } \mathrm{PAH}\end{array}$ & Primary endpoint observed & Models & Reference \\
\hline miR-21 & up-regulated & Increased PASMCs proliferation. & $\begin{array}{l}\text { PASMCs culture from PAH } \\
\text { patients. }\end{array}$ & $\begin{array}{l}\text { Sarkar et al., } \\
2010(70)\end{array}$ \\
\hline miR-98 & down-regulated & Linked with augmented ET-1. & $\begin{array}{l}\text { Pulmonary artery endothelial cell } \\
\text { culture of PAH patients and } \\
\text { hypoxia-induced PAH in rat. }\end{array}$ & $\begin{array}{l}\text { Kang et al., } \\
2016(71)\end{array}$ \\
\hline $\operatorname{miR}-103 / 107$ & down-regulated & $\begin{array}{l}\text { Related to activation of HIF-1 } \\
\text { signaling pathways, enhanced } \\
\text { proliferation of PASMCs and } \\
\text { vascular remodeling in PAH. }\end{array}$ & Hypoxia-induced PAH in rat. & $\begin{array}{c}\text { Deng et al., } \\
2016 \text { (72) }\end{array}$ \\
\hline miR-135a & up-regulated & $\begin{array}{l}\text { High right ventricular systolic } \\
\text { pressure linked to BMPR2 } \\
\text { down-expression in lung. }\end{array}$ & Experimental mouse model. & $\begin{array}{l}\text { Lee and Park, } \\
2017 \text { (75) }\end{array}$ \\
\hline miR-140 & up-regulated & $\begin{array}{l}\text { Linked to reduced MFN1 expression } \\
\text { in hypertrophic right ventricles from } \\
\text { PAH rats. }\end{array}$ & $\begin{array}{l}\text { Sugen-5416 injection plus hypoxia } \\
\text { exposure-induced PAH rats. }\end{array}$ & $\begin{array}{l}\text { Joshi et al., } \\
2016(76)\end{array}$ \\
\hline $\operatorname{miR}-140-5 p$ & down-regulated & $\begin{array}{l}\text { High PASMC proliferation } \\
\text { dependent of SMURF1 pathways. }\end{array}$ & $\begin{array}{l}\text { PASMCs culture from PAH } \\
\text { patients. } \\
\text { Monocrotaline-induced } \mathrm{PAH} \text { in rat. }\end{array}$ & $\begin{array}{l}\text { Rothman et al., } \\
2016(77) ; \\
\text { Zhang and Xu, } \\
2016 \text { (78) }\end{array}$ \\
\hline miR-204 & down-regulated & $\begin{array}{l}\text { RUNX2 overexpression and HIF- } 1 \alpha \\
\text { activation linked to PASMC } \\
\text { proliferation in PAH. }\end{array}$ & $\begin{array}{l}\text { PASMCs culture from PAH } \\
\text { patients, human PAH lung and } \\
\text { Sugen/hypoxia-induced PAH in } \\
\text { rats. }\end{array}$ & $\begin{array}{l}\text { Ruffenach et al., } \\
2016 \text { (79) }\end{array}$ \\
\hline miR-223 & down-regulated & $\begin{array}{l}\text { miR-223 overexpression in hypoxia- } \\
\text { induced PAH model, it is associated } \\
\text { with attenuation in pulmonary arterial } \\
\text { pressure. }\end{array}$ & $\begin{array}{l}\text { Human PAH lung, distal pulmonary } \\
\text { arterioles and PASMCs culture. }\end{array}$ & $\begin{array}{l}\text { Meloche et al., } \\
2015 \text { (82); } \\
\text { Smith et al., } \\
2015 \text { (81) }\end{array}$ \\
\hline miR-210 & up-regulated & $\begin{array}{l}\text { Induce PASMC proliferation HIF-1 } 1 \alpha \\
\text { dependent. }\end{array}$ & $\begin{array}{l}\text { PASMCs culture and hypoxia- } \\
\text { induced PAH in rat. }\end{array}$ & $\begin{array}{l}\text { Gou et al., } \\
2012(87)\end{array}$ \\
\hline $\operatorname{miR}-199 a-5 p$ & up-regulated & $\begin{array}{l}\text { Associated with lower level of oxide } \\
\text { nitric in } \mathrm{PAH} \text {. }\end{array}$ & $\begin{array}{l}\mathrm{PAH} \text { in rat models and PASMCs } \\
\text { culture from PAH patients. }\end{array}$ & $\begin{array}{l}\text { Liu et al., } \\
2016(86)\end{array}$ \\
\hline $\begin{array}{l}\text { let-7a-5p, } \\
\text { miR-26b-5p, } \\
\text { miR-27b-3p, } \\
\text { miR-199a-3p, } \\
\text { and miR-656 }\end{array}$ & up-regulated & $\begin{array}{l}\text { Activated a wide-ranging of Wnt/ } \\
\beta \text {-catenin pathway, leading to } \\
\text { vascular remodeling and } \\
\text { complications in } \mathrm{PAH} \text {. }\end{array}$ & Lung tissues from IPAH patients. & $\begin{array}{l}\text { Wu et al., } \\
2016(88)\end{array}$ \\
\hline
\end{tabular}

miRNA, miR-: micro-RNAs; PASMC: pulmonary artery smooth muscle cells.

Mechanistically, it has been proposed that miR-223 overexpression could inhibit key regulators of actin dynamics and cell proliferation, such as myosin phosphatase (MYPT1) and RhoB, attenuating vascular remodeling and PAH (67). In agreement with this, recent studies have demonstrated that inhibition of the Rho family protein with the selective Rho inhibitors tipifarnib or fasudil is capable of attenuating ventricular remodeling (83) and preventing development of hypoxia-induced PAH (84).

Shi and colleagues demonstrated that the miR-223 down-regulation observed in $\mathrm{PAH}$ is linked to increased expression of the insulin-like growth factor-I receptor (IGF-IR) in human pulmonary hypertension. On the other hand, when miR-223 is overexpressed, or upon pharmacological inhibition of IGF-IR, right-ventricular hypertrophy is attenuated and right heart function is improved under hypoxia (85). Together, these findings show that miR-223 can act in modulating different signaling pathways in $\mathrm{PAH}$.

miRNA-199a-5p is another non-coding RNA that plays important roles in PAH (86). While multiple studies have shown that non-coding RNAs are mostly down-regulated in $\mathrm{PAH}$, a recent study has demonstrated that the expression of miR-199a-5p and miR-210 are significantly increased under PAH $(86,87)$. miR-199a-5p is associated with lower 
levels of $\mathrm{NO}$ in PAH and administration of anti-miR-199a-5p restored increased levels of $\mathrm{NO}$ and improved pulmonary artery pressure and right ventricular hypertrophy (86).

In a study on microRNA abundance in end-stage iPAH, five miRNAs (let-7a-5p, miR-26b-5p, miR-27b-3p, miR-199a-3p, and miR-656) were found to be significantly up-regulated in lung tissues (88). In this study, the authors showed that the upregulation of these miRNAs activated the Wnt / $\beta$-catenin pathway, leading to vascular remodeling and complications of iPAH.

Taken together, these findings have demonstrated that miRNAs could become important biomarkers for the diagnosis of severity and prognosis of $\mathrm{PAH}$. miRNAs also represent a promising target in clinical strategies aiming at improving the prevention and therapeutic treatment of $\mathrm{PAH}$. However, it should be noted that the significance of miRNA expression must be interpreted cautiously depending on the expression site or cells.

\section{Perspectives for treatment and prevention of PAH}

The number of studies examining epigenetic alterations in PAH has been steadily increasing recently. These experimental and clinical studies have demonstrated that epigenetic processes, such as DNA methylation, ubiquitination, miRNAs-dependent gene regulation, and HDACs are related to $\mathrm{PAH}$ (Figure 1), as shown in different animal models. In addition, some reports have demonstrated that restoration of miRNA expression and HDACs inhibitors can be used to attenuate or reverse pathophysiological dysfunction of PAH. Despite the recent advances in the epigenetic field, the identification of a clinical epigenetic therapy with effective reversibility or cure for $\mathrm{PAH}$ is still a challenge for future research. A clinically relevant point raised by Wang and colleagues is that the majority of studies on animal models do not evaluate in depth the disadvantages or collateral effects provoked by epigenetic treatment (89), and initial preclinical studies with epigenetic modifiers have failed to show clinical significance. These issues should be the focus of future investigations.

In addition, more studies examining the epigenetic pathway alterations underlying endothelial dysfunction will be necessary. In brief, it has been demonstrated that epigenetic factors, such as hypermethylation in HDAC4, HDAC5, and HDAC6 gene promoters, down-regulation in miR424/ 503, up-regulation of miR21, miR143, miR210, miR27a, and miR130/301, and upregulation of ion channels could display a potential function in molecular pathways alterations implicated in endothelial dysfunction in PAH (90).

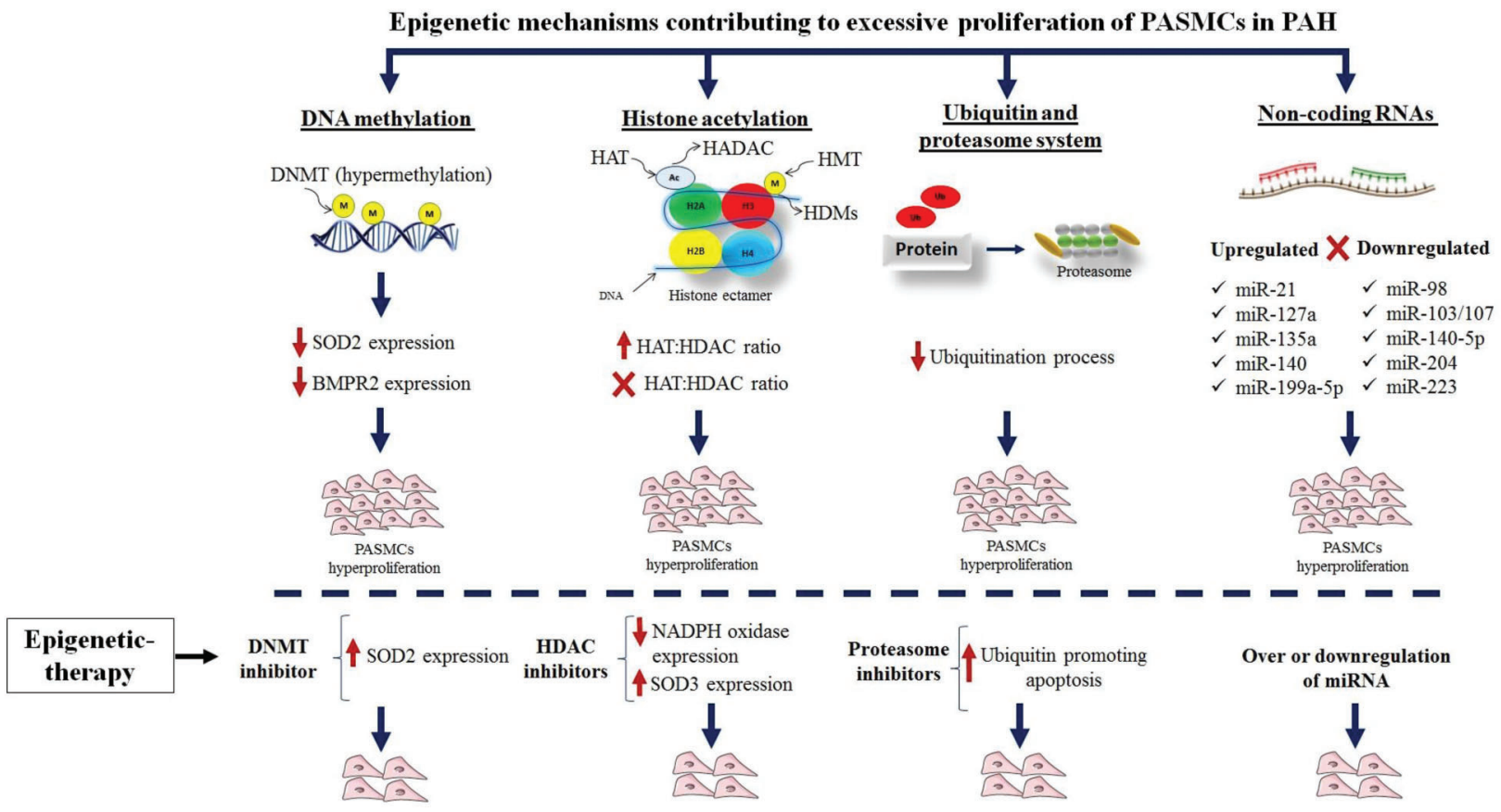

Figure 1. Epigenetic mechanisms contributing to excessive proliferation and resistance to apoptosis of pulmonary artery smooth muscle cells (PASMCs) in pulmonary arterial hypertension (PAH) include: i) DNA methylation via DNA methyltransferases (DNMTs); ii) histone modifications, mainly methylation and acetylation, regulated by histone acetyltransferases (HATs) histone methyltransfereses (HMTs), histone deacetylases (HDACs), and histone demethylases (HDMs). Dysregulation of the ubiquitination process, as well as of microRNAs (miRNA, miR-) also participates in the pathogenesis of PAH. Epigenetic therapy based on DNMT inhibitors, HADAC inhibitors, proteasome inhibitors, and miR-RNA modulators are able to reduce the PASMCs proliferative state in PAH. 
Lastly, effective prevention of PAH needs to be investigated in future research. Experimental protocols examining the epigenetic process in the pre-pathological state of $\mathrm{PAH}$ will help to elucidate possible prevention strategies. For example, it is still unclear whether epigenetic modifications could be preceding the clinical manifestations of $\mathrm{PAH}$. Monocrotaline-induced pulmonary arterial hypertension models in rodents have been shown to exhibit pathophysiological dysfunction after 28 days of intervention. Perhaps, future epigenetic experiments conducted at 14 days in these models will shed some light on the

\section{References}

1. Humbert M, Lau EM, Montani D, Jais $X$, Sitbon $O$, Simonneau G. Advances in therapeutic interventions for patients with pulmonary arterial hypertension. Circulation 2014; 130: 2189-2208, doi: 10.1161/CIRCULATIONAHA. 114.006974.

2. Thenappan T, Ormiston ML, Ryan JJ, Archer SL. Pulmonary arterial hypertension: pathogenesis and clinical management. BMJ 2018; 360: j5492, doi: 10.1136/bmj.j5492.

3. Humbert M, Sitbon O, Chaouat A, Bertocchi M, Habib G, Gressin V, et al. Pulmonary arterial hypertension in France: results from a national registry. Am J Respir Crit Care Med 2006; 173: 1023-1030, doi: 10.1164/rccm.200510-16680C.

4. Thenappan T, Shah SJ, Rich S, Gomberg-Maitland M. A USA-based registry for pulmonary arterial hypertension: 1982-2006. Eur Respir J 2007; 30: 1103-1110, doi: 10.1183/ 09031936.00042107.

5. McGoon MD, Benza RL, Escribano-Subias P, Jiang X, Miller DP, Peacock AJ, et al. Pulmonary arterial hypertension: epidemiology and registries. J Am Coll Cardiol 2013; 62: D51-D59, doi: 10.1016/j.jacc.2013.10.023.

6. Saco TV, Parthasarathy PT, Cho Y, Lockey RF, Kolliputi N. Role of epigenetics in pulmonary hypertension. Am J Physiol Cell Physiol 2014; 306: C1101-C1105, doi: 10.1152/ajpcell. 00314.2013.

7. Kim JD, Lee A, Choi J, Park Y, Kang H, Chang W, et al. Epigenetic modulation as a therapeutic approach for pulmonary arterial hypertension. Exp Mol Med 2015; 47: e175, doi: 10.1038/emm.2015.45.

8. Vaillancourt M, Ruffenach G, Meloche J, Bonnet S. Adaptation and remodelling of the pulmonary circulation in pulmonary hypertension. Can J Cardiol 2015; 31: 407-415, doi: 10.1016/j.cjca.2014.10.023.

9. Wojtala M, Pirola L, Balcerczyk A. Modulation of the vascular endothelium functioning by dietary components, the role of epigenetics. Biofactors 2017; 43: 5-16, doi: 10.1002/biof.1306.

10. Archer SL, Marsboom G, Kim GH, Zhang HJ, Toth PT, Svensson EC, et al. Epigenetic attenuation of mitochondrial superoxide dismutase 2 in pulmonary arterial hypertension: a basis for excessive cell proliferation and a new therapeutic target. Circulation 2010; 121: 2661-2671, doi: 10.1161/ CIRCULATIONAHA.109.916098.

11. Huston JH, Ryan JJ. The emerging role of epigenetics in pulmonary arterial hypertension: an important avenue for clinical trials (2015 Grover Conference Series). Pulm Circ 2016; 6: 274-284, doi: 10.1086/687765. understanding of the pre-pathological epigenetic events before $\mathrm{PAH}$.

\section{Conclusion}

DNMT, HDAC, proteasome inhibitors, and up- or down-regulation of miRNAs can be useful as therapeutic targets in PAH treatment. Modulations of target epigenetic mechanisms are able to reduce the PASMCs proliferative state in PAH and consequently improve right ventricular systolic pressure and right ventricular hypertrophy.

12. Ataya A, Patel S, Cope J, Alnuaimat H. Pulmonary arterial hypertension and associated conditions. Dis Mon 2016; 62: 379-402, doi: 10.1016/j.disamonth.2016.03.006.

13. Yuan JX, Aldinger AM, Juhaszova M, Wang J, Conte JV Jr, Gaine SP, et al. Dysfunctional voltage-gated $\mathrm{K}+$ channels in pulmonary artery smooth muscle cells of patients with primary pulmonary hypertension. Circulation 1998; 98: 1400-1406, doi: 10.1161/01.CIR.98.14.1400.

14. Zhang H, Wang D, Li M, Plecita-Hlavata L, D'Alessandro A, Tauber J, et al. Metabolic and proliferative state of vascular adventitial fibroblasts in pulmonary hypertension is regulated through a MicroRNA-124/PTBP1 (Polypyrimidine tract binding protein 1)/pyruvate kinase muscle axis. Circulation 2017; 136: 2468-2485, doi: 10.1161/CIRCULATIONAHA. 117.028069.

15. Eckberg DL, Drabinsky M, Braunwald E. Defective cardiac parasympathetic control in patients with heart disease. $N$ Engl J Med 1971; 285: 877-883, doi: 10.1056/NEJM 197110142851602

16. da Silva Goncalves Bos D, Van Der Bruggen CEE, Kurakula $K$, Sun XQ, Casali KR, Casali AG, et al. Contribution of impaired parasympathetic activity to right ventricular dysfunction and pulmonary vascular remodeling in pulmonary arterial hypertension. Circulation 2018; 137: 910-924, doi: 10.1161/CIRCULATIONAHA.117.027451.

17. Liu RX, Luo Q, Qiao H, Yu J, Zhang QL, Wang P, et al. Clinical significance of the sympathetic nervous system in the development and progression of pulmonary arterial hypertension. Curr Neurovasc Res 2017; 14: 190-198, doi: $10.2174 / 1567202614666170112165927$.

18. Nootens M, Kaufmann E, Rector T, Toher C, Judd D, Francis GS, et al. Neurohormonal activation in patients with right ventricular failure from pulmonary hypertension: relation to hemodynamic variables and endothelin levels. J Am Coll Cardiol 1995; 26: 1581-1585, doi: 10.1016/0735-1097(95) 00399-1.

19. Mam V, Tanbe AF, Vitali SH, Arons E, Christou HA, Khalil RA. Impaired vasoconstriction and nitric oxide-mediated relaxation in pulmonary arteries of hypoxia- and monocrotaline-induced pulmonary hypertensive rats. J Pharmacol Exp Ther 2010; 332: 455-462, doi: 10.1124/jpet.109.160119.

20. Noble D. Conrad Waddington and the origin of epigenetics. J Exp Biol 2015; 218: 816-818, doi: 10.1242/jeb.120071.

21. de Brito Alves JL, Costa-Silva JH. Maternal protein malnutrition induced-hypertension: New evidence about the autonomic 
and respiratory dysfunctions and epigenetic mechanisms. Clin Exp Pharmacol Physiol 2018; 45: 422-429, doi: 10.1111/ 1440-1681.12892.

22. Huang JB, Liang J, Zhao XF, Wu WS, Zhang F. Epigenetics: novel mechanism of pulmonary hypertension. Lung 2013; 191: 601-610, doi: 10.1007/s00408-013-9505-1.

23. Marchal C, Miotto B. Emerging concept in DNA methylation: role of transcription factors in shaping DNA methylation patterns. J Cell Physiol 2015; 230: 743-751, doi: 10.1002/ jcp. 24836 .

24. Turner BM. Cellular memory and the histone code. Cell 2002; 111: 285-291, doi: 10.1016/S0092-8674(02)01080-2.

25. Fritz JV, Heintz-Buschart A, Ghosal A, Wampach L, Etheridge A, Galas D, et al. Sources and functions of extracellular small RNAs in human circulation. Ann Rev Nutr 2016; 36: 301-336, doi: 10.1146/annurev-nutr-071715-050711.

26. Zhang Y, Ren J. Epigenetics and obesity cardiomyopathy: From pathophysiology to prevention and management. Pharmacol Ther 2016; 161: 52-66, doi: 10.1016/j.pharmthera. 2016.03.005.

27. Rae W. Indications to epigenetic dysfunction in the pathogenesis of common variable immunodeficiency. Arch Immunol Ther Exp 2017; 65: 101-110, doi: 10.1007/s00005-016-0414-x.

28. Haggarty P. Genetic and metabolic determinants of human epigenetic variation. Curr Opin Clin Nutr Metab Care 2015; 18: 334-338, doi: 10.1097/MCO.0000000000000194.

29. Peterson CL, Laniel MA. Histones and histone modifications. Curr Biol 2004; 14: R546-R551, doi: 10.1016/j.cub. 2004.07.007.

30. Jenuwein T. Re-SET-ting heterochromatin by histone methyltransferases. Trends Cell Biol 2001; 11: 266-273, doi: 10.1016/ S0962-8924(01)02001-3.

31. Glozak MA, Sengupta N, Zhang X, Seto E. Acetylation and deacetylation of non-histone proteins. Gene 2005; 363: 15-23, doi: 10.1016/j.gene.2005.09.010.

32. Thiagalingam $\mathrm{S}$, Cheng $\mathrm{KH}$, Lee $\mathrm{HJ}$, Mineva $\mathrm{N}$, Thiagalingam A, Ponte JF. Histone deacetylases: unique players in shaping the epigenetic histone code. Ann N Y Acad Sci 2003; 983: 84-100, doi: 10.1111/j.1749-6632.2003.tb05964.x.

33. Balcerczyk A, Pirola L. Therapeutic potential of activators and inhibitors of sirtuins. Biofactors 2010; 36: 383-393, doi: 10.1002/biof.112.

34. Bedford MT, Richard S. Arginine methylation an emerging regulator of protein function. Mol Cell 2005; 18: 263-272, doi: 10.1016/j.molcel.2005.04.003.

35. Rice JC, Allis CD. Code of silence. Nature 2001; 414: 258-261, doi: 10.1038/35104721.

36. Jahan S, Davie JR. Protein arginine methyltransferases (PRMTs): role in chromatin organization. Adv Biol Regul 2015; 57: 173-184, doi: 10.1016/j.jbior.2014.09.003.

37. Volkel P, Angrand PO. The control of histone lysine methylation in epigenetic regulation. Biochimie 2007; 89: 1-20, doi: 10.1016/j.biochi.2006.07.009.

38. Shi Y, Lan F, Matson C, Mulligan P, Whetstine JR, Cole PA, et al. Histone demethylation mediated by the nuclear amine oxidase homolog LSD1. Cell 2004; 119: 941-953, doi: 10.1016/ j.cell.2004.12.012.

39. Willis MS, Townley-Tilson WH, Kang EY, Homeister JW, Patterson C. Sent to destroy: the ubiquitin proteasome system regulates cell signaling and protein quality control in cardiovascular development and disease. Circ Res 2010; 106: 463-478, doi: 10.1161/CIRCRESAHA.109.208801.

40. Nguyen LK, Dobrzynski M, Fey D, Kholodenko BN. Polyubiquitin chain assembly and organization determine the dynamics of protein activation and degradation. Front Physiol 2014; 5: 4, doi: 10.3389/fphys.2014.00004.

41. Elia L, Condorelli G. RNA (Epi)genetics in cardiovascular diseases. J Mol Cell Cardiol 2015; 89: 11-16, doi: 10.1016/ j.yjmcc.2015.07.012.

42. Archer SL. Acquired mitochondrial abnormalities, including epigenetic inhibition of superoxide dismutase 2, in pulmonary hypertension and cancer: therapeutic implications. Adv Exp Med Biol 2016; 903: 29-53, doi: 10.1007/978-1-48997678-9.

43. Wang J, Zhang C, Liu C, Wang W, Zhang N, Hadadi C, et al. Functional mutations in $5^{\prime}$ UTR of the BMPR2 gene identified in Chinese families with pulmonary arterial hypertension. Pulm Circ 2016; 6: 103-108, doi: 10.1086/685078.

44. Xiong J. BMPR2 spruces up the endothelium in pulmonary hypertension. Protein Cell 2015; 6: 703-708, doi: 10.1007/ s13238-015-0208-7.

45. Atkinson C, Stewart S, Upton PD, Machado R, Thomson JR, Trembath RC, et al. Primary pulmonary hypertension is associated with reduced pulmonary vascular expression of type II bone morphogenetic protein receptor. Circulation 2002; 105: 1672-1678, doi: 10.1161/01.CIR.0000012754. 72951.3D.

46. Viales RR, Eichstaedt CA, Ehlken N, Fischer C, Lichtblau M, Grunig E, et al. Mutation in BMPR2 promoter: a 'second hit' for manifestation of pulmonary arterial hypertension? PloS One 2015; 10: e0133042, doi: 10.1371/journal.pone.0133042.

47. Liu D, Yan Y, Chen JW, Yuan P, Wang XJ, Jiang R, et al. Hypermethylation of BMPR2 promoter occurs in the patients with heritable pulmonary arterial hypertension and inhibits BMPR2 expression. Am J Respir Crit Care Med 2017; 196: 925-928, doi: 10.1164/rccm.201611-2273LE.

48. Cavasin MA, Stenmark KR, McKinsey TA. Emerging roles for histone deacetylases in pulmonary hypertension and right ventricular remodeling (2013 Grover Conference series). Pulm Circ 2015; 5: 63-72, doi: 10.1086/679700.

49. Talati M, Seeley E, Ihida-Stansbury K, Delisser H, McDonald $\mathrm{H}$, Ye F, et al. Altered expression of nuclear and cytoplasmic histone $\mathrm{H} 1$ in pulmonary artery and pulmonary artery smooth muscle cells in patients with IPAH. Pulm Circ 2012; 2: 340-351, doi: 10.4103/2045-8932.101645.

50. Mumby S, Gambaryan N, Meng C, Perros F, Humbert M, Wort SJ, et al. Bromodomain and extra-terminal protein mimic JQ1 decreases inflammation in human vascular endothelial cells: Implications for pulmonary arterial hypertension. Respirology 2017; 22: 157-164, doi: 10.1111/resp. 12872.

51. Price LC, Caramori G, Perros F, Meng C, Gambaryan N, Dorfmuller $\mathrm{P}$, et al. Nuclear factor kappa-B is activated in the pulmonary vessels of patients with end-stage idiopathic pulmonary arterial hypertension. PloS One 2013; 8: e75415, doi: 10.1371/journal.pone.0075415.

52. Demarco VG, Whaley-Connell AT, Sowers JR, Habibi J, Dellsperger KC. Contribution of oxidative stress to pulmonary arterial hypertension. World J Cardiol 2010; 2: 316-324, doi: 10.4330/wjc.v2.i10.316. 
53. Chen F, Li X, Aquadro E, Haigh S, Zhou J, Stepp DW, et al. Inhibition of histone deacetylase reduces transcription of NADPH oxidases and ROS production and ameliorates pulmonary arterial hypertension. Free Radic Biol Med 2016; 99: 167-178, doi: 10.1016/j.freeradbiomed.2016.08.003.

54. Masri FA, Comhair SA, Dostanic-Larson I, Kaneko FT, Dweik RA, Arroliga AC, et al. Deficiency of lung antioxidants in idiopathic pulmonary arterial hypertension. Clin Trans/ Sci 2008; 1: 99-106, doi: 10.1111/j.1752-8062.2008.00035.x.

55. Kamezaki F, Tasaki H, Yamashita K, Tsutsui M, Koide S, Nakata S, et al. Gene transfer of extracellular superoxide dismutase ameliorates pulmonary hypertension in rats. $A m \mathrm{~J}$ Resp Crit Care Med 2008; 177: 219-226, doi: 10.1164/ rccm.200702-264OC

56. Nozik-Grayck E, Woods C, Stearman RS, Venkataraman S, Ferguson BS, Swain K, et al. Histone deacetylation contributes to low extracellular superoxide dismutase expression in human idiopathic pulmonary arterial hypertension. Am J Physiol Lung Cell Mol Physiol 2016; 311: L124-L134, doi: 10.1152/ajplung.00263.2015.

57. Lan B, Hayama E, Kawaguchi N, Furutani Y, Nakanishi T. Therapeutic efficacy of valproic acid in a combined monocrotaline and chronic hypoxia rat model of severe pulmonary hypertension. PloS One 2015; 10: e0117211, doi: 10.1371/ journal.pone.0117211.

58. Li M, Dong X, Liu Y, Sun X, Li Z, He J. Inhibition of ubiquitin proteasome function suppresses proliferation of pulmonary artery smooth muscle cells. Naunyn Schmiedebergs Arch Pharmacol 2011; 384: 517-523, doi: 10.1007/s00210-0110678-y.

59. Ibrahim YF, Wong CM, Pavlickova L, Liu L, Trasar L, Bansal $G$, et al. Mechanism of the susceptibility of remodeled pulmonary vessels to drug-induced cell killing. J Am Heart Assoc 2014; 3: e000520, doi: 10.1161/JAHA.113.000520.

60. Jeganathan N, Predescu D, Predescu S. Intersectin-1s deficiency in pulmonary pathogenesis. Respir Res 2017; 18 : 168, doi: 10.1186/s12931-017-0652-4.

61. Kim SY, Lee JH, Huh JW, Kim HJ, Park MK, Ro JY, et al. Bortezomib alleviates experimental pulmonary arterial hypertension. Am J Respir Cell Mol Biol 2012; 47: 698708, doi: 10.1165/rcmb.2011-03310C.

62. Wang X, Ibrahim YF, Das D, Zungu-Edmondson M, Shults NV, Suzuki YJ. Carfilzomib reverses pulmonary arterial hypertension. Cardiovas Res 2016; 110: 188-199, doi: 10.1093/ $\mathrm{cvr} / \mathrm{crw} 047$

63. Leopold JA, Maron BA. Molecular mechanisms of pulmonary vascular remodeling in pulmonary arterial hypertension. Intl J Mol Sci 2016; 17. pii: E761, doi: 10.3390/ijms170 50761.

64. Guo L, Yang Y, Liu J, Wang L, Li J, Wang Y, et al. Differentially expressed plasma microRNAs and the potential regulatory function of Let-7b in chronic thromboembolic pulmonary hypertension. PloS One 2014; 9: e101055, doi: $10.1371 /$ journal.pone.0101055.

65. Bienertova-Vasku J, Novak J, Vasku A. MicroRNAs in pulmonary arterial hypertension: pathogenesis, diagnosis and treatment. J Am Soc Hypertens 2015; 9: 221-234, doi: 10.1016/j.jash.2014.12.011.

66. Croce CM, Calin GA. miRNAs, cancer, and stem cell division. Cell 2005; 122: 6-7, doi: 10.1016/j.cell.2005.06.036.
67. Zeng Y, Zhang X, Kang K, Chen J, Wu Z, Huang J, et al. MicroRNA-223 attenuates hypoxia-induced vascular remodeling by targeting RhoB/MLC2 in pulmonary arterial smooth muscle cells. Sci Rep 2016; 6: 24900, doi: 10.1038/srep 24900.

68. Elia L, Condorelli G. MicroRNAs and pulmonary hypertension: a tight link. Cardiovas Res 2016; 111: 163-164, doi: $10.1093 / c v r / c v w 163$.

69. Yuan K, Orcholski M, Tian X, Liao X, de Jesus Perez VA. MicroRNAs: promising therapeutic targets for the treatment of pulmonary arterial hypertension. Expert Opinion Therap Targets 2013; 17: 557-564, doi: 10.1517/14728222.2013. 765863.

70. Sarkar J, Gou D, Turaka P, Viktorova E, Ramchandran R, Raj JU. MicroRNA-21 plays a role in hypoxia-mediated pulmonary artery smooth muscle cell proliferation and migration. Am J Physiol Lung Cell Mol Physiol 2010; 299: L861-L871, doi: 10.1152/ajplung.00201.2010.

71. Kang BY, Park KK, Kleinhenz JM, Murphy TC, Green DE, Bijli KM, et al. Peroxisome Proliferator-Activated Receptor gamma and microRNA 98 in Hypoxia-Induced Endothelin-1 Signaling. Am J Resp Cell Mol Biol 2016; 54: 136-146, doi: 10.1165/rcmb.2014-03370C.

72. Deng B, Du J, Hu R, Wang AP, Wu WH, Hu CP, et al. MicroRNA-103/107 is involved in hypoxia-induced proliferation of pulmonary arterial smooth muscle cells by targeting HIF-1beta. Life Sci 2016; 147: 117-124, doi: 10.1016/j.Ifs. 2016.01.043.

73. Kang BY, Park KK, Green DE, Bijli KM, Searles CD, Sutliff $\mathrm{RL}$, et al. Hypoxia mediates mutual repression between microRNA-27a and PPARgamma in the pulmonary vasculature. PloS One 2013; 8: e79503, doi: 10.1371/journal.pone. 0079503.

74. Xie X, Li S, Zhu Y, Liu L, Pan Y, Wang J, et al. MicroRNA27a/b mediates endothelin-1-induced PPARgamma reduction and proliferation of pulmonary artery smooth muscle cells. Cell Tissue Res 2017; 369: 527-539, doi: 10.1007/ s00441-017-2625-9.

75. Lee HW, Park SH. Elevated microRNA-135a is associated with pulmonary arterial hypertension in experimental mouse model. Oncotarget 2017; 8: 35609-35618, doi: 10.18632/ oncotarget.16011.

76. Joshi SR, Dhagia V, Gairhe S, Edwards JG, McMurtry IF, Gupte SA. MicroRNA-140 is elevated and mitofusin- 1 is downregulated in the right ventricle of the Sugen5416/ hypoxia/normoxia model of pulmonary arterial hypertension. Am J Physiol Heart Circ Physiol 2016; 311: H689-H698, doi: 10.1152/ajpheart.00264.2016.

77. Rothman AM, Arnold ND, Pickworth JA, Iremonger J, Ciuclan L, Allen RM, et al. MicroRNA-140-5p and SMURF1 regulate pulmonary arterial hypertension. J Clin Invest 2016; 126: 2495-2508, doi: 10.1172/JCl83361.

78. Zhang $\mathrm{Y}, \mathrm{Xu}$ J. MiR-140-5p regulates hypoxia-mediated human pulmonary artery smooth muscle cell proliferation, apoptosis and differentiation by targeting Dnmt1 and promoting SOD2 expression. Bioch Biophys Res Commun 2016; 473: 342-348, doi: 10.1016/j.bbrc.2016.03.116.

79. Ruffenach G, Chabot S, Tanguay VF, Courboulin A, Boucherat O, Potus F, et al. Role for Runt-related Transcription Factor 2 in Proliferative and Calcified Vascular Lesions 
in Pulmonary Arterial Hypertension. Am J Respir Crit Care Med 2016; 194: 1273-1285, doi: 10.1164/rccm.201512-23800C

80. Chen CZ, Li L, Lodish HF, Bartel DP. MicroRNAs modulate hematopoietic lineage differentiation. Science 2004; 303: 83-86, doi: 10.1126/science.1091903.

81. Smith KA, Yuan JX, Schumacker PT. MicroRNAs and PARP: co-conspirators with ROS in pulmonary hypertension. Focus on "miR-223 reverses experimental pulmonary arterial hypertension". Am J Physiol Heart Circ Physiol 2015; 309: C361-C362, doi: 10.1152/ajpcell.00209.2015.

82. Meloche J, Le Guen M, Potus F, Vinck J, Ranchoux B, Johnson I, et al. miR-223 reverses experimental pulmonary arterial hypertension. Am J Physiol Heart Circ Physiol 2015; 309: C363-C372, doi: 10.1152/ajpcell.00149.2015.

83. Wang ZH, Zhu D, Xie S, Deng Y, Pan Y, Ren J, et al. Inhibition of Rho-kinase attenuates left ventricular remodeling caused by chronic intermittent hypoxia in rats via suppressing myocardial inflammation and apoptosis. J Cardiovas Pharmacol 2017; 70: 102-109, doi: 10.1097/FJC.000000 0000000496.

84. Duluc L, Ahmetaj-Shala B, Mitchell J, Abdul-Salam VB, Mahomed AS, Aldabbous L, et al. Tipifarnib prevents development of hypoxia-induced pulmonary hypertension. Cardiovas Res 2017; 113 276-287, doi: 10.1093/cvr/cvw258.

85. Shi L, Kojonazarov B, Elgheznawy A, Popp R, Dahal BK, Bohm M, et al. miR-223-IGF-IR signalling in hypoxia- and load-induced right-ventricular failure: a novel therapeutic approach. Cardiovas Res 2016; 111: 184-193, doi: 10.1093/ cvr/crw065

86. Liu Y, Liu G, Zhang H, Wang J. MiRNA-199a-5p influences pulmonary artery hypertension via downregulating Smad3. Biochem Biophys Res Commun 2016; 473: 859-866, doi: 10.1016/j.bbrc.2016.03.140.

87. Gou D, Ramchandran R, Peng X, Yao L, Kang K, Sarkar J, et al. miR-210 has an antiapoptotic effect in pulmonary artery smooth muscle cells during hypoxia. Am J Physiol Lung Cell Mol Physiol 2012; 303: L682-L691, doi: 10.1152/ ajplung.00344.2011

88. Wu D, Talbot CC Jr, Liu Q, Jing ZC, Damico RL, Tuder R, et al. Identifying microRNAs targeting Wnt/beta-catenin pathway in end-stage idiopathic pulmonary arterial hypertension. J Mol Med (Berl) 2016; 94: 875-885, doi: 10.1007/ s00109-016-1426-z.

89. Wang Y, Yan L, Zhang Z, Prado E, Fu L, Xu X, et al. Epigenetic regulation and its therapeutic potential in pulmonary hypertension. Front Pharmacol 2018; 9: 241, doi: 10.3389/ fphar.2018.00241.

90. Ranchoux B, Harvey LD, Ayon RJ, Babicheva A, Bonnet S, Chan SY, et al. Endothelial dysfunction in pulmonary arterial hypertension: an evolving landscape (2017 Grover Conference Series). Pulm Circ 2018; 8: 2045893217752912 , doi: $10.1177 / 2045893217752912$ 